\title{
Effectiveness of physical, social and digital mechanisms against laptop theft in open organizations $^{1}$
}

\author{
Trajce Dimkov, Wolter Pieters, Pieter Hartel \\ Distributed and Embedded Security Group \\ University of Twente, The Netherlands \\ $\{$ trajce.dimkov, wolter.pieters, pieter.hartel\}@utwente.nl
}

\begin{abstract}
Organizations rely on physical, digital and social mechanisms to protect their IT systems. Of all IT systems, laptops are probably the most troublesome to protect, since they are easy to remove and conceal. When the thief has physical possession of the laptop, it is also difficult to protect the data inside. In this study, we look at the effectiveness of the security mechanisms against laptop theft in two universities. The study considers the physical and social protection of the laptops. We analyze the logs from laptop thefts in both universities and complement the results with penetration tests. The results from the study show that the effectiveness of security mechanisms from the physical domain is limited, and it depends mostly from the social domain. The study serves as a motivation to further investigate the analysis of the alignment of the mechanisms across all three security domains to protect the IT assets in an organization.
\end{abstract}

Keywords: laptop theft, case study, physical security, digital security, social engineering.

\section{INTRODUCTION}

Of all IT systems, laptops are particularly hard to protect. Laptops are mobile, easily concealable, there is a big market to sell the hardware and there can be many of them in a single building. With the increased data storage capabilities of laptops, the loss of even a single laptop can induce dramatical costs to the organization [2]. Thus, although there can be a large number of laptops in an organization, losing even a single laptop may not be acceptable.

Organizations open to the public are particularly at risk from laptop theft. Hospitals and universities, for example, accept hundreds of people that can wander in the premises every day. Marshall et al. [3] points out that $46 \%$ of data breaches occur in institutions open to the public: education, health care and the government. Laptops containing sensitive medical or academic data become highly vulnerable in these environments.

The problem security professionals face is how to protect the laptops in such open organizations. There are three types of security mechanisms to secure laptops in a building: digital, physical and social mechanisms. Digital mechanisms such as laptop tracking and remote data deletion protect the

${ }^{1}$ A poster containing partial results of the study was presented at CCS[1].

This research is supported by the Sentinels program of the Technology Foundation STW, applied science division of NWO and the technology programme of the Ministry of Economic Affairs under project number TIT.7628. laptop and the data in the laptop by using software. Physical mechanisms, such as doors and cameras, physically isolate the thief from the laptop and/or identify her in case of a theft. Social mechanisms such as organizational policies and rules decrease the number of mistakes by employees and increase the resilience of employees toward social engineering. Using digital mechanisms to protect laptops is elaborately researched by the computer science community $[4,5,6,7]$. However, linking these mechanisms with physical and social mechanisms in protecting laptops is still not explored.

This paper evaluates the existing physical and social security mechanisms for protecting laptops based on (1) logs of laptop thefts which occurred in a period of two years in two universities in Netherlands, and (2) 14 penetration tests in the same universities. The goal of the penetration tests was to gain possession of a marked laptop from an employee unaware of the penetration test. The results from the log analysis and the penetration tests show that the security of an asset in an open organization depends mainly on the level of security awareness of the employees, and to a lesser extent on the technical or physical security mechanisms. The physical and technical mechanisms have a passive, deterrent role on reducing theft, while the employees have an active, preventive role.

The outline of the rest of the paper is as follows. In section 2 we provide a literature overview on laptop theft. In section 3 we evaluate the logs of the laptop thefts and in section 4 we describe the penetration tests and the results from the tests. Section 5 summarizes our conclusions and suggests a guideline for which mechanisms should be considered first in adding security mechanisms. Section 6 concludes the paper.

\section{LiterATURE OVERVIEW}

There are two areas of research that focus on protecting laptops: computer science and crime science.

In the computer science community, there has been a considerable effort to model the complex security relations between the digital, physical and social domain. Scott et al. $[8,9]$ provides a holistic security model of the world by using spatial relationship between the elements in the ambient calculus [10]. Dragovic et al. [11, 12] presents a model which uses the physical property of objects and the sensitivity of the data inside the objects to identify possible threats. In 
our previous work [13], we also provided a formal model for representing and analysis of policy misalignment between the three domains. These models provide sound policy design but do not ensure effectiveness of security mechanisms that enforce these policies.

There are multiple mechanisms in computer science that work either in the physical or digital domain. In the digital domain, several security products, such as TrueCrypt ${ }^{1}$ and BitLocker ${ }^{2}$ provide encryption for the whole hard drive. These solutions assume the adversary does not have physical control of the laptop, because if the adversary has physical possession of the laptop, she can always successfully execute a number of attacks $[14,15,16]$. These approaches also seem to ignore the human element, or more precisely, induce performance overhead and decrease the usability of the laptop. A recent study by Panemon [17] shows that the majority of non-IT individuals, even when provided with an encrypted laptop, turn off the encryption software.

A number of tracking applications, such as Adeona [7] and LoJack [5], can track the location of the laptop they are installed on. In case of theft, these solutions use the Internet to provide the owner with the current location of the laptop. These solutions suffer from two problems: (1) if the goal of the theft is obtaining data from the laptop, the thief might never connect the laptop to Internet and (2) if the goal is to obtain the hardware, the thief can easily remove the tracking application by flashing the BIOS and/or formatting the hard drive.

In the computer science community, the accent is on protecting the data residing in the laptop and finding the location of the stolen laptop. The approach from the crime science community is more general, and considers the laptop and its environment. The goal in this field is to prevent a thief from stealing the laptop in the first place, by either changing the environment surrounding the laptop or by creating situations that will deter a thief [18]. Kitteringham [19] provides a list of 117 strategies how to prevent laptop theft. The strategies include the implementation of physical, digital and social mechanisms. Although the list is elaborate, all suggested mechanisms focus only on a single domain and do not consider any interaction between the mechanisms.

There are several other studies that analyze laptop theft. These reports focus on the money loss from a stolen laptop [2] and the frequency of laptop theft and the most affected sectors [3]. Our results are complementary, and look at the effectiveness of conventional physical and social security mechanisms in stopping laptop theft.

\section{Methodology}

Assessing the effectiveness of a security mechanism can be achieved by auditing and penetration testing. We apply both methodologies to investigate the most commonly used security systems in the physical and social domain.

\footnotetext{
${ }^{1}$ www.truecrypt.org

${ }^{2}$ blogs.technet.com/bitlocker
}

First, we look at logs of recent laptop thefts in two universities in Netherlands. From the logs we obtain information about: the last control that failed before the laptop theft, alarms raised by the theft and the role of physical mechanisms in securing the laptop and finding the thief, such as access control and surveillance cameras. The logs provide valuable information on the approaches thieves use to steal a laptop. However, the logs provide limited information about the level of security awareness of the employees. In particular, the logs do not provide any information on the possible violation of social security mechanisms, such as letting strangers inside an office and sharing credentials between employees. Even in case of a burglary, the logs did not provide any information how the thief reached the burgled office. Therefore, to better understand the effect of the security mechanisms, we orchestrated 14 penetration tests where we used social engineering to steal a laptop. Through the tests, we observed the security awareness of the employees as well as the efficiency of the physical security mechanisms in both universities.

\section{A. Log analysis}

In a period of two years, the two universities suffered from 59 laptop thefts (Figure 1). The logs from the thefts provide (1) the location from where the laptop was stolen, (2) protection mechanisms on the laptop, and (3) how the theft was discovered.

1) Location of the theft: In $30 \%$ of the thefts, the thief broke into a locked office either by forcing the door or breaking a window. This number indicates failure of the physical security mechanisms in both campuses. In $46 \%$ of the thefts, the laptop was stolen when the employee left it unattended in a public location, such as a cafeteria or meeting room. These thefts indicate the level of security awareness of the employees. In $19 \%$ of the cases, the theft occurred when the employee left the office for a short period of time without locking the door. These results show a combined failure of the physical and social mechanisms. The low security awareness let the users leave the laptops unattended in a restricted area, and the physical security mechanisms did not protect the laptops from being stolen.

2) Protection mechanisms on the laptop: In five of the thefts that occurred in an unlocked office, the laptop was locked with Kensington lock. Only one of the laptops stolen in a public location was locked with a Kensington lock.

3) Theft discovery: The majority of the thefts (93\%) were reported by the laptop owner. In a few cases the report came from an employee who observed a broken door or window $(5 \%)$. Only one of the thefts triggered an alarm. In this case, the thief grabbed the laptop while the employee went to collect print outs and left through the fire door, triggering the fire alarm. In all buildings, in both universities, there are surveillance cameras (CCTV) and either partially or fully centralized access control systems able to log access requests. Surprisingly, the systems provided no useful information in any of the thefts. These mechanisms are further analyzed in section IV. 


\begin{tabular}{||l||c|c|c|c|c||c||}
\hline \hline & $\begin{array}{c}\text { Locked } \\
\text { office } \\
\text { (burglary) }\end{array}$ & $\begin{array}{c}\text { Open } \\
\text { office }\end{array}$ & $\begin{array}{c}\text { Restricted } \\
\text { location }\end{array}$ & $\begin{array}{c}\text { Public } \\
\text { location }\end{array}$ & $\begin{array}{c}\text { No } \\
\text { details }\end{array}$ & Total \\
\hline \hline Stolen laptops & 18 & 11 & 2 & 27 & 1 & 59 \\
\hline Cut Kensington locks & 1 & 5 & 0 & 1 & 0 & 7 \\
\hline Other physical damage & 16 & 0 & 0 & 0 & 0 & 16 \\
\hline \hline
\end{tabular}

Figure 1. Information from the logs. The logs from both universities are merged to anonymize the data.

4) Limitation of the logs: The logs provide information obtained after the theft took place, based on evidence found by the police and the security guards. The logs provide information only of successful theft attempts, but do not show when the security controls have succeeded in stopping the thief. Moreover, the logs do not provide information on how the thief reached the location nor on whether the security awareness of the employees contributed to the theft.

Researchers and organizations recognize that the employees are the weakest link in the organization [20, 21, 22]. Since the logs from the laptop thefts were insufficient to provide us with this information, we orchestrated a set of penetration tests where we used social engineering as a means to obtain a laptop.

\section{B. The penetration tests}

To perform the penetration tests, we enlisted the help from 45 master students in computer security who took the role of penetration testers. Before performing the tests we informed and received permission for the penetration tests from the chief security officers in both universities. We informed the officers exactly which locations we were going to test and the names of the staff and students involved. No other security person in the universities knew of the tests. The tests were approved by the legal department from the universities.

The students were divided in teams of three. The goal of each team was to steal a clearly marked laptop from an employee who was unaware of the penetration test. First, we did a pilot study with only three teams and three laptops. Based on the results and insights of the pilot study, we performed an additional 11 penetration tests the next year.

The rest of the section defines (1) the roles in a penetration test, (2) the setup, (3) the execution and (4) the closure phase in the test, and discusses (5) the results and (6) the limitations of the tests.

1) Roles in the penetration test: We define five roles in the penetration tests.

1 Coordinator - an employee responsible for the experiment and the behavior of the penetration tester. The coordinator orchestrates the penetration tests.

2 Penetration tester - a student who attempts to gain possession of the asset without being caught.

3 Contact person - an employee who volunteers to distribute the asset to the custodians.

4 Custodian - an employee at whose office the laptop is placed.
5 Employee - person in the university who has none of the roles above.

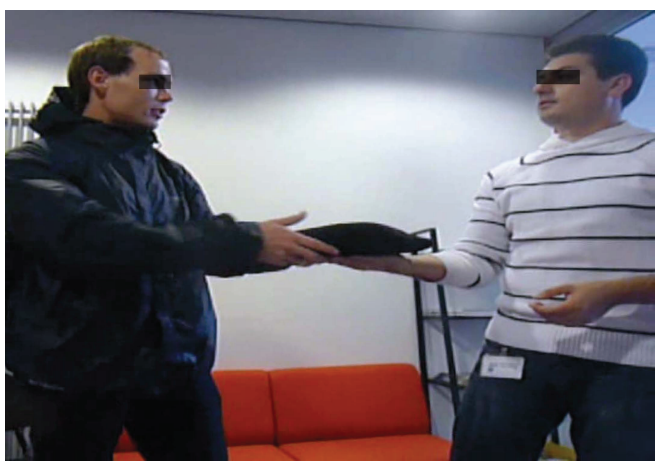

Figure 2. In nine of the tests the custodians willingly gave the laptop, either believing that the teams were from the help desk or that they were sent by the coordinator.

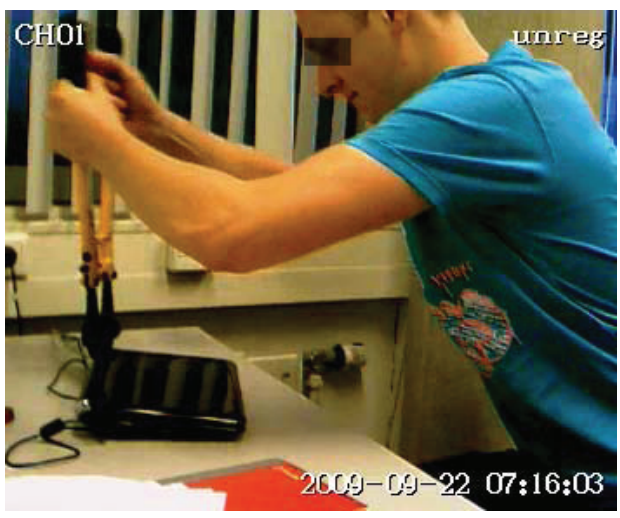

Figure 3. In five tests the teams social engineered a person other than the custodian. In two of these cases the students used a bolt cutter to cut the Kensington lock, and in three cases they found the keys from the lock in the office.

2) Setup of the environment: At the start of the study we used snowball sampling [23] to recruit a group of contact persons and custodians. We chose four contact persons, who in turn searched for other volunteers willing to take part in the study as custodians.

After selecting the contact people and the custodians, we bought and marked 14 laptops as assets to be stolen. The contact persons asked the custodians to sign an informed consent form, and then distributed the clearly marked laptops, each with a web-camera and a Kensington lock. The custodians resided in two different universities in nine different buildings. 
To steal any of the laptops, the penetration testers needed to circumvent at least three layers of access control: the entrance of the building, the entrance of the office where the custodian works and the Kensington lock.

To avoid bias in the study, none of the custodians was aware of the real purpose of the study. Instead, we informed the custodians that the universities were conducting a usability study on the new laptops, and thus they needed to measure the satisfaction level of the laptop users. We informed the custodians that the level of satisfaction would be measured using motion detection web-cameras that would record the usage of the laptops. Furthermore, for security reasons, the contact people instructed the custodians to lock the laptops with a Kensington lock and to leave the cameras recording at all times. The contact people also asked the custodians not to leave any private nor work related data on the laptops. With these measures, we tried to reduce the risk of data leakage and loss of productivity caused by any theft. More detailed analysis of the methodology and the motivation behind the decision design is presented in [24].

3) Execution of the penetration tests: After setting up the environment, we gave each of the penetration teams the location of a single laptop they should obtain. In the first part, each team scouted their location and collected as much information as possible about the custodian and the security mechanisms at the location. Then, each team proposed a list of attack scenarios they wanted to conduct. A sample attack scenario is presented in Figure 4. During the second part of the test, after getting approval for executing the scenarios by the coordinator, the teams started testing.

The actions of the teams were logged using the web-cameras we positioned in the offices of the custodians and through recording devices carried by the teams during the attacks. We used such comprehensive recordings (1) to have a better overview of why the attacks succeeded/failed and (2) to be sure the employees were treated with respect by the penetration testers. The students were asked to try to avoid the CCTV cameras, to reflect the behavior of a real thief.

After each successful or failed attempt, the teams provided an attack trace listing which mechanisms they circumvented and, in case of failed attempts, which mechanism caused the attack to fail. Figure 5 provides a summary of the successful approaches of teams and the disguises they used to obtain the laptop.

4) Closure: After all penetration tests were over, we debriefed the custodians and the contact people through a group presentation, where we explained the penetration test and its goal. All custodians and contact people were thanked and rewarded for helping in the assessment of the security in their university.

5) Results: Surprisingly, all teams were eventually successful in stealing their laptop. Besides the 14 successful thefts, there were an additional 11 unsuccessful attempts.

The favorite approach of the teams was to confront the custodian directly and ask for the laptop. Nine of the teams took roles as service desk employees, students that urgently
1. Social engineer night pass from an employee.

2. Enter the building early in the morning.

3. Social engineer the cleaning lady to access the office.

4. Cut any protection on the laptop using a bolt cutter.

5. Leave the building during office hours.

Figure 4. Example of an attack scenario

needed a laptop for a few hours or claimed that they were sent by the coordinator. Four teams used mobile phones or pocket video cameras to record the conversation with the employees. In one case they took a professional camera and a cameraman, and told the custodian the recording is part of a study to measure the service quality of the service desk.

\begin{tabular}{|c|c|c|}
\hline Approach & Disguise & \\
\hline Social engineered the custodian & as coordinator helpers & 5 \\
\hline & as help desk & 2 \\
\hline & as students & 2 \\
\hline Social engineered the janitor & as students & 4 \\
\hline Social engineered the cleaning lady & as PhD student & 1 \\
\hline
\end{tabular}

Figure 5. Approaches and disguises of the penetration testing teams

The resistance of the employees against social engineering varied. In six cases, the custodians gave the laptop easily after being shown a fake email and being promised they would get the laptop back in a few hours. In two cases the custodian wanted a confirmation from the coordinator. The teams succeeded in the attempt because the custodian called a number provided by the penetration testers. Needless to say, the number was of another team member pretending to be the coordinator. In one case a colleague of the custodian got suspicious and sent an email to campus security. Since only the chief security officer knew about the penetration test, in a few hours the security guards all over the campus were all alerted and started searching for suspicious students.

However, in five cases the students were not able to social engineer the custodian directly and were forced to look for alternative approaches. For example, in one of the cases the students entered the building before working hours. At this time a cleaning lady cleaned the offices, and under the assumption it was their office let the students inside. After entering the office, the students cut the Kensington lock and left the building before the custodian arrived. On the way out, they even asked the same cleaning lady to lock again the office door.

6) Limitations of the test: A limitation of the test might be the high self-confidence of the testers. The security guards were not aware of the penetration test. If caught, the identification process would be unpleasant experience for the testers. Nevertheless, they knew they will not go to jail for their actions. A thief might rather wait for the laptop to be left unattended than approaching an employee directly and asking for their laptop.

\section{OBSERVATIONS}

We observed three main security mechanisms in the universities: surveillance cameras, access control and a level of 
security awareness of the employees.

\section{A. Surveillance cameras}

Security officers do not use cameras as alarming mechanisms, but use recorded footages a posteriori, to identify an offender after an accident has taken place. The security officers cannot afford to monitor all surveillance cameras. The cameras work only when a motion is detected, and automatically store the recording in a back end server. The delay between the occurrence and report of the theft gives the thief sufficient time to leave the building.

Even when used to identify the thief a posteriori, the cameras provide limited information about the thief. In none of the logs nor during any of the penetration tests the cameras provided enough information to reveal the identity of the thief. The CCTV system is providing limited help because (1) the cameras are not mounted in offices, (2) the thief can easily conceal the laptop and (3) thieves usually know the position of the cameras and obscure their face.

The cameras are not mounted in offices. All penetration tests and $49 \%$ of the thefts took place in an office. Cameras are not mounted in offices to preserve the privacy of the employees and because mounting cameras in every office is not cost effective. Without surveillance in these offices, it is impossible to identify a thief during the act.

Instead of offices, the cameras are usually mounted on the entrance of buildings. Many people pass through the entrances with bags, and each of the bags might conceal a stolen laptop. Even if there are only two persons observed by the camera, if the persons are not caught on the spot and challenged by the security guards, the evidence from the surveillance camera can not be used against them.

Cameras positioned to monitor public locations, such as cafeterias, halls and reception desks can record the thief during the theft. The logs show that $46 \%$ of the laptop thefts happened in public locations. During the penetration tests we noticed that these cameras are usually triggered by motion detection, and are not actively monitored by the security guards. A careful thief would obscure her face from the cameras using a hat, a hood or just covering her face with her hands before she steals the laptop. In one of the penetration tests, three penetration testers wandered with newspapers on top of their faces through the building without being challenged by anybody.

In conclusion, the surveillance system provides no help in stopping the theft and has limited usage in identifying the thief a posteriori.

\section{B. Access control}

We spotted two weaknesses of the access control in the universities. Locks are usually bypassed because (1) they are disabled during working hours and (2) the doors and windows where the locks reside are easy to force.

The access controls on the entrances of the building are easily bypassed because they are disabled during working hours and because there are too many people with credentials that can open the door. From the 14 penetration teams, 13 bypassed the entrance locks by attacking during working hours and one team social engineered credentials from an employee to enter the building out of working hours.

Another attack vector for stealing a laptop is to force a door or a window. The penetration teams were not allowed to damage any property of the universities except cutting the Kensington locks. However, the logs from actual laptop thefts show that in $30 \%$ of the thefts, the thief broke a door or a window to get access to the office.

Similarly to recordings from surveillance cameras, logs from the access control systems provide limited help in identifying the thief. The logs show whose credential was used to enter a restricted area at a specific time period. Since the credentials are easy to steal or social engineer and because there are many people entering and leaving the area where the theft occurs, it is hard to deduce which person is the thief.

In conclusion, the typical access control mechanisms deployed in the universities are mainly used to deter opportunistic thieves, but provide no help against a determined thief.

\section{Security awareness of the employees}

The level of security awareness of the employees plays a crucial role in success or failure of a theft. The human element is the main reason behind the success of the laptop thefts. In $69 \%$ of the laptop thefts and $100 \%$ of the penetration tests, the theft occurred either because the employee left the laptop unattended in a public location or did not lock the door when leaving the office. Similarly, during the penetration tests, employees opened door from offices of their colleagues, shared credentials or handed in laptops without any identification. Therefore, even with strong access control in place, if the security awareness of the employees is low, the access control can easily be circumvented.

On the other hand, the human element is the main reason behind the failure of $67 \%$ of all failed penetration tests. In these cases, an employee informed the security guards for suspicious activities, rejected to open a door for the tester, rejected to unlock a laptop without permission from the custodian or interrupted the tester during the theft. In these cases, the employees besides enforcing the access control mechanisms, also played a role as an additional surveillance layer around the laptop.

Employees are usually considered as the weakest link in the security of an organization. We observe that employees can also be the strongest link in the security of open organization. A proper security education of employees increases the employee's resistance to social engineering, and increases effectiveness of the other security mechanisms.

\section{Limitations of the observations}

The observations from the test and log analysis is based on the security mechanisms in two open institutions. The observations may apply to other mobile assets, such as medical equipment, beamers and mobile phones in institutions open to the public. However, other types of organizations might have different spectrum of mechanisms for protecting their laptops. 


\section{CONCLUSiON}

In this paper we evaluated the security mechanisms from the physical and social domain that influence laptop theft in organizations open to the public. We analyzed the logs of laptop thefts which occurred in a period of two years in two universities in Netherlands. We complemented the findings from these logs with 14 penetration tests, in which we used social engineering to gain possession of marked laptops.

We observed that (1) mechanisms from a single domain can provide only limited protection against laptop and (2) the effectiveness of a physical security mechanism depends mainly from its alignment with security mechanisms from social domain.

From this study we identify two research questions:

1) How to formally analyze the alignment of the mechanisms from all three domains and find possible threats that lead to laptop theft?

2) How to test the implementation of the mechanisms using all actions available to the thief: digital penetration testing, obtaining physical access and social engineering?

In the future we will focus on three areas: First we plan to expand this exploratory study by performing another series of penetration tests to obtain statistically significant results. Secondly, we intend to build upon our previous work in analysis of security policies to provide designs which enhance the effectiveness of the security mechanisms. Finally, we will focus on developing better methodologies for penetration testing where testers can use physical access and social engineering. Such methodologies should provide reliable and reportable results from testing the implementation of the mechanisms.

\section{REFERENCES}

[1] T. Dimkov, W. Pieters, and P. Hartel. Laptop theft: a case study on the effectiveness of security mechanisms in open organizations. In CCS '10: Computer and Communications Security, pages 666-668, NY, USA, 2010. ACM.

[2] L. Ponemon. Cost of a lost laptop. Technical report, Ponemon Institute, 2009. communities.intel.com/docs/DOC-3076.

[3] M. Marshall, M. Martindale, R. Leaning, and D. Das. Data Loss Barometer. KPMG, UK, 2008. www.datalossbarometer.com.

[4] Seagate Technology. Can your computer keep a secret? 2007.

[5] Absolute Software. Lojack for laptops www.lojackforlaptops.com.

[6] Wayne A. Jansen, Serban I. Gavrila, and Vlad Korolev. Proximity-based authentication for mobile devices. In Security and Management, pages 398-404, 2005.

[7] T. Ristenpart, G. Maganis, A. Krishnamurthy, and T. Kohno. Privacy-preserving location tracking of lost or stolen devices: cryptographic techniques and replacing trusted third parties with dhts. In SS'08, pages 275-290, Berkeley, CA, USA, 2008. USENIX Association.
[8] D.J. Scott. Abstracting Application-Level Security Policy for Ubiquitous Computing. $\mathrm{PhD}$ thesis, University of Cambridge, 2004.

[9] D.J. Scott, A. Beresford, and A. Mycroft. Spatial policies for sentient mobile applications. Policies for Distributed Systems and Networks, pages 147-157, 2003.

[10] L. Cardelli and A.D. Gordon. Mobile ambients. Theoretical Computer Science, 240(1):177-213, 2000.

[11] B. Dragovic and J. Crowcroft. Information exposure control through data manipulation for ubiquitous computing. In NSPW '04: Proceedings of the 2004 workshop on New security paradigms, pages 57-64. ACM, 2004.

[12] B. Dragovic and J. Crowcroft. Containment: from context awareness to contextual effects awareness. In Proceedings of 2nd Inernational Workshop on Software Aspects of Context. CEUR Workshop Proceedings, 2005.

[13] T Dimkov, W. Pieters, and Hartel P. Portunes: representing attack scenarios spanning through the physical, digital and social domain. In ARSPA-WITS, 2010.

[14] P. Kleissner. Stoned bootkit. In Black Hat USA, 2009.

[15] E.M. Chan, J.C. Carlyle, F.M. David, R. Farivar, and R.H. Campbell. Bootjacker: compromising computers using forced restarts. In CCS '08: 15th ACM conference on Computer and communications security, pages 555-564, NY, USA, 2008. ACM.

[16] S. Türpe, A. Poller, J. Steffan, J.P. Stotz, and J. Trukenmüller. Attacking the bitlocker boot process. In Trust '09: Proceedings of the 2nd International Conference on Trusted Computing, pages 183-196, Berlin, Heidelberg, 2009. Springer-Verlag.

[17] L. Ponemon. The human factor in laptop encryption. Technical report, Ponemon Institute, December 2008.

[18] D.B. Cornish and R.V. Clarke. Opportunities, precipitators and criminal decisions: A reply to Wortley's critique of situational crime prevention. Crime Prevention Studies, 16:41-96, 2003.

[19] G. Kitteringham. Lost laptops = lost data: Measuring costs, managing threats. Crisp report, ASIS International Foundation, 2008.

[20] N. Barrett. Penetration testing and social engineering hacking the weakest link. Information Security Technical Report, 8(4):56-64, 2003.

[21] T. Ashish. Social engineering: An attack vector most intricate to tackle. Technical report, Infosecwriters, 2007.

[22] M. Workman. Gaining access with social engineering: An empirical study of the threat. Information Security Journal: A Global Perspective, 16(6):315-331, 2007.

[23] B.L.A. Goodman. Snowball sampling. The Annals of Mathematical Statistics, 32(1):148-170, 1961.

[24] T. Dimkov, W. Pieters, and P. Hartel. Two methodologies for physical penetration testing using social engineering. In $A C S A C$ '10, Chicago, USA, 2010. ACM. 